\title{
Magnetic ordering in the spinel compound $\mathrm{Li}\left[\mathrm{Mn}_{2-x} \mathrm{Li}_{x}\right] \mathrm{O}_{4}(x=0,0.04)$
}

\author{
John Gaddy, ${ }^{1}$ Jagat Lamsal, ${ }^{1}$ Marcus Petrovic, ${ }^{1}$ Wouter Montfrooij, ${ }^{1, a)}$ \\ Alexander Schmets, ${ }^{2}$ and Thomas Vojta ${ }^{3}$ \\ ${ }^{1}$ Department of Physics and Missouri Research Reactor, University of Missouri, Columbia, \\ Missouri 65211, USA \\ ${ }^{2}$ Reactor Institute Delft, Technical University of Delft, 2629 JB Delft, The Netherlands \\ ${ }^{3}$ Department of Physics, Missouri University of Science and Technology, Rolla, Missouri 65409, USA
}

(Presented 12 November 2008; received 16 September 2008; accepted 1 December 2008; published online 11 March 2009)

\begin{abstract}
The two $B$-site ions $\mathrm{Mn}^{3+}$ and $\mathrm{Mn}^{4+}$ in the stoichiometric spinel structure $\mathrm{LiMn}_{2} \mathrm{O}_{4}$ form a complex, columnar ordered pattern below the charge-ordering transition at room temperature. On further cooling to below $66 \mathrm{~K}$, the system develops long-range antiferromagnetic order. In contrast, whereas lithium-substituted $\mathrm{Li}\left[\mathrm{Mn}_{2-x} \mathrm{Li}_{x}\right] \mathrm{O}_{4}$ also undergoes a charge-ordering transition around room temperature, it only displays frozen in short-range magnetic order below $\sim 25-30 \mathrm{~K}$. We investigate to what extent the columnar charge-order pattern observed in $\mathrm{LiMn}_{2} \mathrm{O}_{4}$ can account for the measured magnetic ordering patterns in both the pure and Li-substituted $(x=0.04)$ compounds. We conclude that eightfold rings of $\mathrm{Mn}^{4+}$ ions form the main magnetic unit in both compounds $(x=0,0.04)$, and that clusters formed out of these rings act as superspins in the doped compound.

(C) 2009 American Institute of Physics. [DOI: 10.1063/1.3073660]
\end{abstract}

The ground state properties of the known lithium-based cubic spinel compounds $\mathrm{LiT}_{2} \mathrm{O}_{4}$ range from $\mathrm{BCS}$ superconductivity $^{1}[T=\mathrm{Ti}]$, via heavy fermion behavior ${ }^{2}[T$ $=\mathrm{V}]$ to frustrated antiferromagnetism ${ }^{3,4}[T=\mathrm{Mn}]$. In these systems, the divalent $B$-site ions (3+ and $4+$ ) have an octahedral oxygen surrounding, while the $\mathrm{Li}^{+}$ions occupy the $A$-sites. In this paper we focus on the magnetism in the $\mathrm{Mn}$ compound. Above $\sim 300 \mathrm{~K}, \mathrm{LiMn}_{2} \mathrm{O}_{4}$ is an electronhopping conductor, and the system undergoes a chargeordering (CO) transition on cooling down. Similar to the CO transition in magnetite, ${ }^{5}$ the $B$-site octahedra are slightly deformed and a structural transition accompanies the $\mathrm{CO}$ transition. However, unlike for magnetite, the $\mathrm{Mn}^{3+}-\mathrm{Mn}^{4+}$ charge-ordered structure has (most likely) been resolved by Rodriguez-Carvajal et al. ${ }^{6}$

In stoichiometric $\mathrm{LiMn}_{2} \mathrm{O}_{4}$ the $\mathrm{Mn}^{3+}$ ions line up in columns along the $c$-axis ${ }^{6}$ when cooled to below $300 \mathrm{~K}$, see Fig. 1(a). Multiple types of $\mathrm{Mn}^{3+}$ sites can be distinguished. One type is located in cubes of four $\mathrm{Mn}^{3+}$ ions and four $\mathrm{O}^{2-}$ ions $(\mathrm{Mn}-\mathrm{O}$ distance $=2.05 \AA)$ stacked along the $c$-direction. These cubes are at the center of eightfold rings of $\mathrm{Mn}^{4+}$ ions. The $\mathrm{Mn}^{4+}$ ions within these rings couple antiferromagnetically (AF) to each other through a $90^{\circ} \mathrm{Mn}-\mathrm{O}-\mathrm{Mn}$ exchange. The spaces in between neighboring rings are filled with the other types of $\mathrm{Mn}^{3+}$ ions, which thus form columns in the $c$-direction. The unit cell $(a=24.74 \AA, b=24.84 \AA$, and $c$ $=8.20 \AA$ ) houses eight eightfold rings. ${ }^{6}$ The $\mathrm{Mn}^{4+}$ rings only interact with other rings via the intervening $\mathrm{Mn}^{3+}$ ions. When cooled to below $66 \mathrm{~K}$, AF ordering develops, ${ }^{7}$ but the ordered structure has not been resolved yet.

The Li-doped material $\mathrm{Li}\left[\mathrm{Mn}_{2-x} \mathrm{Li}_{x}\right] \mathrm{O}_{4}$ has also been studied in detail ${ }^{3,4}$ because of its applications as a battery material. ${ }^{8}$ When a small amount of $\mathrm{Li}$ is substituted on the

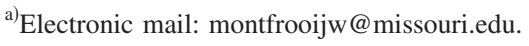

Mn sites, the material retains its capacity for removal of $\mathrm{Li}$ from the $A$-sites without affecting the overall spinel structure (hence its use in lithium batteries), but the $\sim 300 \mathrm{~K}$ structural phase transition no longer takes place, even though the $\mathrm{CO}$ transition is unaffected. (Suppression of the structural transition greatly enhances the lifetime of the battery material during charging/discharging cycles. ${ }^{8}$ ) Neutron scattering studies $^{3}$ on the doped material have shown that long-range magnetic order no longer takes place, but instead the material appears to enter a spin glass phase around $25-30 \mathrm{~K}$ (depending on the exact amount of Li substitution).

Schimmel et al. ${ }^{4}$ described the short-range order in the glass phase of $\mathrm{Li}\left[\mathrm{Mn}_{2-x} \mathrm{Li}_{x}\right] \mathrm{O}_{4}(x=0.04)$ by a network of nonlinear $\mathrm{Mn}^{4+}$ chains, with an average correlation length of
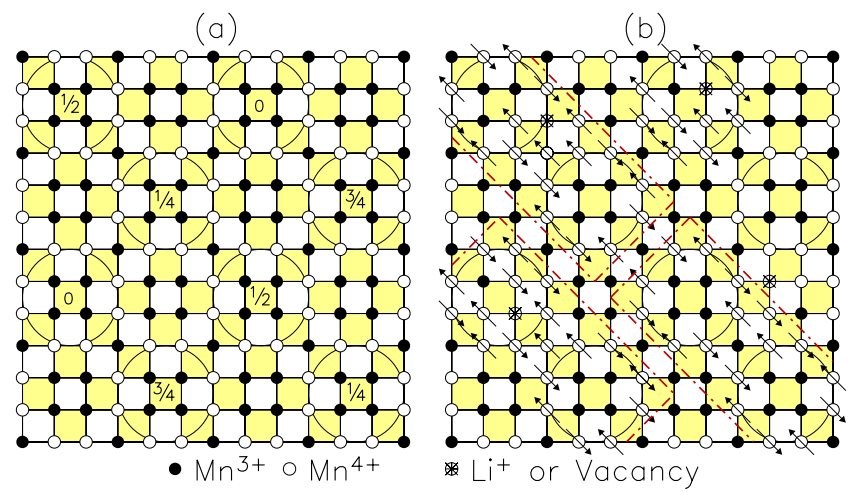

FIG. 1. (Color online) The proposed (Ref. 6) charge-ordered pattern for $\mathrm{LiMn}_{2} \mathrm{O}_{4}$. The figures show a projection down the $c$-axis. (a) The $\mathrm{Mn}^{4+}$ ions form eightfold rings, and the $\mathrm{Mn}^{3+}$ ions are located in between the rings and inside of the rings. Shaded cubes signify lattice positions where the Mn ions interact through the $\mathrm{Mn}-\mathrm{O}-\mathrm{Mn}$ exchange interaction. The numbers inside the rings show their $z$ position. (b) Upon Li substitution on the Mn sites or Mn removal, some $\mathrm{Mn}^{3+}$ ions will become $\mathrm{Mn}^{4+}$ ions, leading to modified rings and linked rings, some possibilities of which are shown. The dashed lines delineate the clusters. 


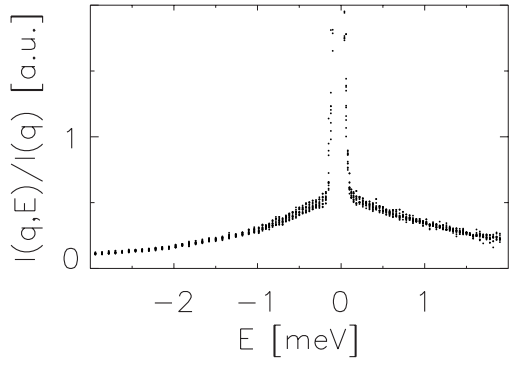

FIG. 2. The energy dependence of the scattered intensity $I(q, E)$ for 12 $q$-values $0.8<q<1.9 \AA^{-1}$ at $T=50 \mathrm{~K}$ (Ref. 4). The energy resolution is given by the sharp central line due to incoherent nuclear scattering. After normalizing to $I(q)=\int_{0.6}^{1} I(q, E) d E$ (Ref. 9), all data points collapse onto a single curve, showing that the time scale of the dynamics is independent of $q$. The figure contains 1600 independent data points.

about four to five ions. While this description gives a satisfactory description of the observed elastic scattering, it is not consistent with the level of Li doping, nor does it explain the dynamics. As to the former objection, only 1 in $50 \mathrm{Mn}^{4+}$ ions will be substituted with Li for $x=0.04$, and even the inclusion of vacancies should leave chains well in excess of 25 members. As to the latter, if the structural units were indeed that small, we should expect to see a difference in the quasielastic scattering between when the units are probed on a length scale smaller than or larger than their size $L$, around $q=2 \pi / L \sim 1.2 \AA^{-1}$. However, the quasielastic scattering ${ }^{4,9}$ displays no length scale dependence, as shown in Fig. 2. Instead, the scattering is what would be expected of much larger structural units that are fully aligned within the units, but that behave as superspins when it comes to unit-to-unit interaction. Therefore, it is very likely that structural units are present in the glass phase that are much larger than clusters of four to five $\mathrm{Mn}^{4+}$ ions.

In this paper we investigate whether the ordering details of this glass phase can be linked to the Rodriguez-Carvajal (RC) structure for undoped $\mathrm{LiMn}_{2} \mathrm{O}_{4}$ (Fig. 1). We argue that the magnetic ordering in $\mathrm{Li}\left[\mathrm{Mn}_{2-x} \mathrm{Li}_{x}\right] \mathrm{O}_{4}$ is indeed closely linked to the $\mathrm{CO}$ pattern in the parent compound. At $\sim 66 \mathrm{~K}$ elastic magnetic scattering by the $\mathrm{Mn}^{4+}$ ions starts to develop ${ }^{4}$ in the doped compound, which turns out to be associated with the eightfold rings. We argue that these ring clusters act as AF superspins: The moments within a cluster become fully AF-aligned below $66 \mathrm{~K}$, but they can all flip in unison similar to the more familiar ferromagnetic superspin. Below $\sim 25-30 \mathrm{~K}$ this superspin flipping becomes so slow ${ }^{4}$ that these clusters resemble static clusters without long-range order between them. We discuss these claims in the following.

Inelastic neutron scattering experiments showed that the dynamics associated with $\mathrm{Mn}^{4+}$ ions is much slower than that of the $\mathrm{Mn}^{3+}$ ions, and freezes out below 25-30 K. ${ }^{4}$ The scattering associated with the $\mathrm{Mn}^{3+}$ ions shows spin reorientations down to the lowest temperatures even though some $\mathrm{Mn}^{3+}$ scattering becomes elastic. Thus, the $\mathrm{Mn}^{4+}-\mathrm{Mn}^{4+} \mathrm{AF}$ interaction must be stronger than the $\mathrm{Mn}^{4+}-\mathrm{Mn}^{3+}$ and the $\mathrm{Mn}^{3+}-\mathrm{Mn}^{3+}$ interactions. Based on this alone, it would make sense for the $\mathrm{Mn}^{4+}$ ions in the eightfold clusters to line up at some finite temperature. From the ordering transition in the

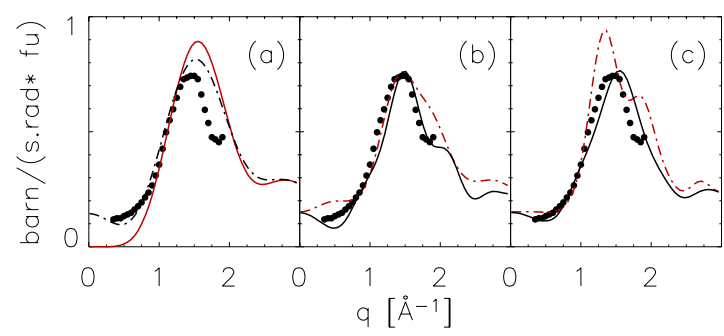

FIG. 3. (Color online) The observed scattering in $\mathrm{Li}\left[\mathrm{Mn}_{2-x} \mathrm{Li}_{x}\right] \mathrm{O}_{4}$ at $30 \mathrm{~K}$ (Ref. 4) (circles). (a) A threefold cluster of $\mathrm{Mn}^{4+}$ ions (dashed line) or an eightfold ring cluster (solid line) fail to capture the width of the observed scattering. (b) Clusters consisting of two rings linked along the $c$-direction (solid line, top right cluster in Fig. 1(b) where only half of it is shown) or along the $a b$-direction [dashed line, top left cluster in Fig. 1(b)] capture most of the observed scattering when the Mn spins are aligned in the $a b$-plane. (c) same as (b), but with the spins aligned along the $c$-direction.

undoped compound, ${ }^{7}$ the temperature at which this happens is apparently $66 \mathrm{~K}$.

The doping with $\mathrm{Li}$ on the $\mathrm{Mn}$ sites changes $\mathrm{Mn}^{3+}$ ions into $\mathrm{Mn}^{4+}$. In a unit cell that has $144 \mathrm{Mn}$ ions (Fig. 1) the Li substitution is about 1:50, or roughly three $\mathrm{Mn}$ atoms per unit cell. As a direct consequence, about eight $\mathrm{Mn}^{3+}$ ions will change to $\mathrm{Mn}^{4+}$. This in turn leads to a unit cell that now has ring clusters of 7, 8, and 9 members [Fig. 1(b)], as well as larger clusters of 17 members, or perhaps even larger if the substitution resulted in multiple rings being linked. This pattern is further enhanced when vacancies appear on the $\mathrm{Mn}$ sites. In $\mathrm{Li}\left[\mathrm{Mn}_{2-x} \mathrm{Li}_{x}\right] \mathrm{O}_{4}(x=0.04)$ as many as 1:40 Mn sites may be vacant, ${ }^{4}$ and these vacancies have a similar effect on the $\mathrm{Mn}^{3+} / \mathrm{Mn}^{4+}$ ratio as $\mathrm{Li}$ substitution has. Thus, we can expect that most eightfold rings are now linked to others, and that they have either a $\mathrm{Li}$ ion or a vacancy embedded in them.

To check whether this modified ring model can describe the neutron scattering data, we plot the elastic scattering of $\mathrm{Mn}^{4+}$ ions at $30 \mathrm{~K},{ }^{4}$ in Fig. 3 . We chose this temperature as it is very close to the glass transition and well below the $66 \mathrm{~K}$ ordering transition ${ }^{7}$ in undoped $\mathrm{LiMn}_{2} \mathrm{O}_{4}$ so that we can expect the $\mathrm{Mn}^{4+}$ ions within the rings (if present) to have lined up. However, the temperature is still high enough that any spin dynamics associated with the $\mathrm{Mn}^{3+}$ ions should not have frozen out. From the width $\Gamma$ of this scattering we find that the $\mathrm{Mn}^{4+}$ spins are correlated over a distance of $2 \pi / \Gamma$ $\sim 10 \AA^{-1}$. The nonzero level of the scattering at low $q$ is a measure of how many Mn ions are missing (roughly 1 in 8 ).

We compare the measured scattering to the scattering of various clusters in Fig. 3. The agreement between the observed and calculated scattering of two linked rings [Fig. 3 (b)] is encouraging since we expect these to be the dominant clusters provided the RC model is correct, and even more so, there are no adjustable parameters in the comparison. We can also distinguish between a spin orientation along the $c$-axis or in the $a b$-plane, even though the data are powder data. From the difference in agreement between Figs. 3(b) and 3(c), we conclude that the Mn moments lay in the $a b$-plane. The total scattering will be a combination of the clusters shown in Fig. 3(b), in addition to a few remaining untouched eight-ring clusters as well as some clusters that embody more than two rings. Clusters that have more than 
two rings in them are bound to be present; however, single crystal data are required to determine the exact distribution. From the data shown in Fig. 3(b) it would appear that multiple ring clusters linked in the $a b$-plane might be invoked to yield a perfect agreement with the data. We did not pursue this avenue of perfect agreement since this would involve using the position of links between rings as adjustable parameters. Nonetheless, given the level of agreement [Fig. 3 (b)] in combination with the lack of any length scale in the dynamics, we can safely conclude that $\mathrm{Mn}^{4+}$ clusters are indeed present in $\mathrm{Li}\left[\mathrm{Mn}_{2-x} \mathrm{Li}_{x}\right] \mathrm{O}_{4}$, and that these clusters most likely have eightfold rings as their main structural units with the $\mathrm{Mn}$ moments in the $a b$-plane fully lined up within a cluster below $66 \mathrm{~K}$. Between $\sim 25$ and $66 \mathrm{~K}$ the clusters then act as superspins, and the superspins freeze out below $\sim 25-30 \mathrm{~K}$.

Having established the ordering within the $\mathrm{Mn}^{4+}$ clusters, it is interesting to speculate whether the long-range magnetic ordering in pure $\mathrm{LiMn}_{2} \mathrm{O}_{4}$ (Ref. 7) is also mainly associated with the ordering of the $\mathrm{Mn}^{4+}$ ions. If so, then at $66 \mathrm{~K}$ the $\mathrm{Mn}^{4+}$ ions would line up AF within the rings, with long-range order being associated with a particular stacking pattern of these rings in the unit cell. The $\mathrm{Mn}^{3+}$ ions would only act as mediators to establish the order among the rings. We find that this speculation cannot describe all the details of the measured diffraction pattern ${ }^{7}$ even though we find qualitative agreement. First, all but one (a small peak at $0.6 \AA^{-1}$ ) of the magnetic peaks indexes onto the RC-unit cell ${ }^{6}$ they do not index onto a smaller sized unit cell. Second, a stacking pattern wherein neighboring rings line up AF is able to reproduce roughly the intensities of the main peaks, and the absence of many other peaks. However, there is no stacking pattern of the eightfold rings that can reproduce the exact intensities of the allowed reflections. In addition, while there still is diffuse scattering present at $2 \mathrm{~K}^{7}$ this scattering is too weak to account for all the $\mathrm{Mn}^{3+}$ ions. Therefore, it is likely that some $\mathrm{Mn}^{3+}$ ions partake in the ordered structure. At present, we do not know whether these ordered $\mathrm{Mn}^{3+}$ ions would be the $\mathrm{Mn}^{3+}$ cubes within the rings, or the $\mathrm{Mn}^{3+}$ ions located between neighboring rings. Overall, given the partial agreement of a structure consisting of ordered eightfold rings with the observed intensities, and the ability to index the peak positions (and lack thereof) using the RC-unit cell, it is highly likely that $\mathrm{LiMn}_{2} \mathrm{O}_{4}$ has eightfold rings consisting of $\mathrm{AF}$-aligned $\mathrm{Mn}^{4+}$ ions in its structure.

In conclusion, we have shown that the $\mathrm{CO}$ pattern as proposed for $\mathrm{LiMn}_{2} \mathrm{O}_{4}$ survives in $\mathrm{Li}$-doped $\mathrm{Li}\left[\mathrm{Mn}_{2-x} \mathrm{Li}_{x}\right] \mathrm{O}_{4}$. The main energy scale for magnetic ordering is that of the antiferromagnetic $\mathrm{Mn}^{4+}-\mathrm{Mn}^{4+}$ interaction. The $\mathrm{Mn}^{4+}$ ions in the eightfold rings line up below $66 \mathrm{~K}$, and in the undoped sample these rings likely form the backbone of the longrange ordered structure. In the doped sample, doping induced linking between these rings results in clusters that act as superspins, which freeze out below $\sim 25-30 \mathrm{~K}$.

This material is based on work supported by the Department of Energy under Award No. DE-FG02-07ER46381 and by the University of Missouri Research Board (Grant No. RB-07-52).

${ }^{1}$ M. R. Harrison, P. P. Edwards, and J. B. Goodenough, Philos. Mag. B 52, 679 (1985).

${ }^{2}$ S. Kondo, D. C. Johnston, C. A. Swenson, F. Borsa, A. V. Mahajan, L. L. Miller, T. Gu, A. I. Goldman, M. B. Maple, D. A. Gajewski, E. J. Freeman, N. R. Dilley, R. P. Dickey, J. Merrin, K. Kojima, G. M. Luke, Y. J. Uemura, O. Chmaissem, and J. D. Jorgensen, Phys. Rev. Lett. 78, 3729 (1997).

${ }^{3}$ V. W. J. Verhoeven, F. M. Mulder, and I. M. de Schepper, Physica B 276-278, 950 (2000).

${ }^{4}$ G. Schimmel, W. Montfrooij, G. J. Kearley, V. W. J. Verhoeven, and I. M. de Schepper, Phys. Rev. B 63, 214409 (2001).

${ }^{5}$ J. Garcia and G. Subias, J. Phys.: Condens. Matter 16, R145 (2004).

${ }^{6}$ J. Rodriguez-Carvajal, G. Rousse, C. Masquelier, and M. Hervieu, Phys. Rev. Lett. 81, 4660 (1998).

${ }^{7}$ J. E. Greedan, C. R. Wiebe, A. S. Wills, and J. R. Stewart, Phys. Rev. B 65, 184424 (2002).

${ }^{8}$ M. M. Thackeray, J. Am. Ceram. Soc. 82, 3347 (1999).

${ }^{9}$ J. Lamsal, J. Gaddy, M. Petrovic, W. Montfrooij, and T. Vojta, "The search for quantum critical scaling in a classical system," J. Appl. Phys. (these proceedings). 